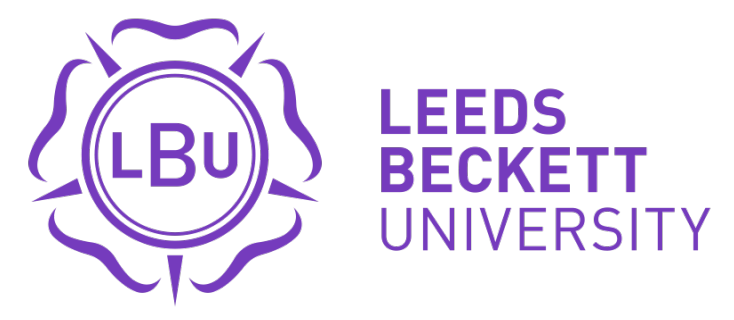

Citation:

Liao, F and Wang, Y and Lu, Y and Deng, J (2017) Optimal preview control for a class of linear continuous-time large-scale systems. Transactions of the Institute of Measurement and Control, 40 (14). pp. 4004-4013. ISSN 0142-3312 DOI: https://doi.org/10.1177/0142331217740946

Link to Leeds Beckett Repository record:

https://eprints.leedsbeckett.ac.uk/id/eprint/5627/

Document Version:

Article (Accepted Version)

The aim of the Leeds Beckett Repository is to provide open access to our research, as required by funder policies and permitted by publishers and copyright law.

The Leeds Beckett repository holds a wide range of publications, each of which has been checked for copyright and the relevant embargo period has been applied by the Research Services team.

We operate on a standard take-down policy. If you are the author or publisher of an output and you would like it removed from the repository, please contact us and we will investigate on a case-by-case basis.

Each thesis in the repository has been cleared where necessary by the author for third party copyright. If you would like a thesis to be removed from the repository or believe there is an issue with copyright, please contact us on openaccess@leedsbeckett.ac.uk and we will investigate on a case-by-case basis. 


\title{
Optimal Preview Control for a Class of Linear Continuous-Time Large-scale
}

\author{
Systems \\ Fucheng Liao ${ }^{\mathrm{a}^{*}}$, Yu Wang ${ }^{\mathrm{a}}$, Yanrong $\mathrm{Lu}^{\mathrm{a}}$ and Jiamei Deng ${ }^{\mathrm{b}}$

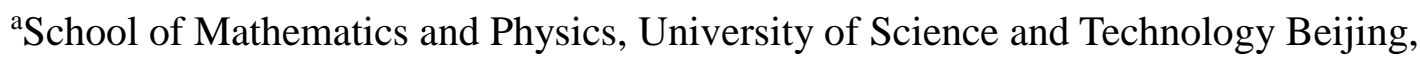 \\ Beijing 100083, China \\ beeds Sustainability Institute, Leeds Beckett University, Leeds, UK LS2 9EN \\ * Corresponding author. Email: fcliao@ustb.edu.cn
}

\begin{abstract}
In this paper, the problem of optimal preview control is studied for a class of linear continuous-time large-scale systems. We first construct an augmented system including the error signal and the reference signal to transform the tracking problem into the regulator problem. Then, the controllers are designed for isolated augmented subsystems, which also constitute the controller of large-scale systems. On the basis of proving the asymptotic stability of closed-loop large-scale systems and the existence of the controller, sufficient conditions for reaching optimal preview control are given. In particular, the limiting condition of the correlation matrices is determined by the fact that the total derivative of a positive definite Lyapunov function is negative definite. The numerical simulation indicates that the controller can drive the large-scale systems to track the reference signal without steady-state error, and the tracking effect is improved with the increasing preview length.
\end{abstract}




\section{Keywords}

Continuous-time large-scale systems, isolated subsystems, asymptotic stability, optimal preview control

\section{Introduction}

Preview control is a control method to improve the tracking accuracy of a system via utilizing the known future information of desired reference signal. The scientific community began a journey for exploring the preview control since Sheridan (1966), who put forward the concept for the first time, and then theoretical results with respect to preview control theory have been formed after more than 50 years of research and development. There are some early efforts such as Bender (1968), Tomizuka (1975), Katayama et al. (1985), Katayama et al. (1987) and Takeshi et al. (1994). Certainly, the preview control for linear systems with constant coefficients was studied thoroughly (Katayama et al., 1985, Katayama et al., 1987, Zhang et al., 1996 and Li et al., 2002), and the robust preview control was also concerned (Tomizuka, 1996 and Lee et al., 1996). In the last decade, when combining with other control theories, preview control was discussed deeply for multi-rate systems (Liao et al., 2003), time-varying systems (Liao et al., 2015), and also descriptor systems (Zhao et al., 2016). Alternatively, some

problems on $H_{\infty}$ optimal preview control were addressed (Kojima, 2015 and Kojima et al., 2003).

The systems with high dimension of state vector are referred to as large-scale 
systems which are characterized by large scale, complex structure, numerous influencing factors and comprehensive functions. Many practical systems, e.g., multiarea power system, coupled water reservoirs, nuclear power systems and processes in the chemical and petroleum industry (see, Lunze, 1992, Michel et al., 1977), can be considered as large-scale systems. A key method for analysis and design of large-scale systems is decomposition-aggregation, which can not only decrease parametric complexity and the computational difficulties that significantly grow as the scale of the systems increase, but also make us have a clear view over the effect of different factors on the behavior of the whole system (see, Araki, 1978). In fact, large-scale systems can be considered as the systems that consist of several isolated subsystems with connection to other subsystems. By devising controller for each isolated subsystem, the controller for the large-scale systems is obtained by integrating subsystem controllers. And if the correlation matrices satisfy certain constraints, the closed-loop large-scale systems would have the required properties (Shi et al., 1992 and Michel et al., 1977). To be more exact, if each of the isolated subsystems is stable, and the norms of the correlation matrices are small enough, then the large-scale systems are stable as well.

Because of the interconnective matrices between subsystems, stability analysis has become one of the most important requirements for large-scale systems. During the past several years, many methods have been established for this issue, such as M-matrix method (Araki, 1978), piecewise Lyapunov method (Zhang et al., 2008), etc. Recently, small gain theorem was used to study robustness problem of large-scale systems. In (Duan et al., 2004), linear matrix inequality conditions were provided for this problem. In (Dashkovskiy et al., 2012, Liu et al., 2011), input-to-state stability Lyapunov functions were constructed for large-scale systems based on small gain theorem. For 
controller design, decentralized control is a main research field of large-scale systems, which is also acknowledge as the effective method to overcome the increasing size and computational complexity of the mathematical models describing large-scale systems. Shi et al. (1992) designed decentralized controller for model-following problem under the scenario that the bounds of the interconnections were known or unknown, respectively. When considering the interconnections between the subsystems as uncertainties, Labibi et al. (2002) designed a decentralized controller for large-scale systems via minimizing weighted sensitivity functions. Based on parameter-dependent Lyapunov function, Duan et al. (2008) utilized linear matrix inequality to devise the decentralized controller that can reduce the design conservation. In (Zhang et al., 2008), the authors considered the problem of $H_{\infty}$ decentralized controller design for discrete-time fuzzy large-scale systems, and analyzed the stability by using piecewise Lyapunov functions.

As pointed out in (Moelja et al., 2006), all or parts of the reference signals are known in advance in certain systems. However, to the best of our knowledge, many controller design methods for trajectory tracking of large-scale systems do not take the preview information into account (see, e.g., Lunze, 1992, Ruan et al., 2005, Ruan et al., 2008). Due to the fact that preview information of reference signal can be applied to improve tracking accuracy effectively, this paper is concerned with the optimal preview tracking control for a class of linear continuous-time large-scale systems. This paper has the following two novel features. The first is to express the reference signal as a new form similar to convex combination, where the number of items in combination is equal to those of subsystems. We view each item in combination as a virtual reference signal, 
then it can be observed from formula (2) that the tracking problem of the whole large-scale system can be converted into several tracking problems of low dimensional subsystems. The second is to exploit decomposition method to obtain isolated subsystems, and meanwhile apply state augmented technique to further translate the tracking problems into optimal regulation problems of several isolated augmented subsystems. When the norms of the associated matrices satisfy certain bounds, the optimal preview controllers are robust to the associated matrices. As a result, the optimal preview controller is designed easily for each of the isolated augmented subsystems by using standard optimal preview control theory, these controllers also constitute the controller of the whole large-scale system. In addition, in order to ensure stability of the whole closed-loop large-scale system, Lyapunov stability theorem and M-matrix is employed to determine the norm bounds of associated matrices. Finally, a numerical example is given to illustrate the effectiveness of the proposed design method.

Notations. $\lambda(A)$ and $\lambda_{\max }(A)$ donate the eigenvalue and the maximum eigenvalue of matrix $A$, respectively. $Q>0 \quad(Q \geq 0)$ donates that symmetric matrix $Q$ is positive definite (positive semi-definite). $I_{n}$ denotes an $n \times n$ identity matrix.

\section{System Description}

\section{Consider linear continuous-time large-scale systems}




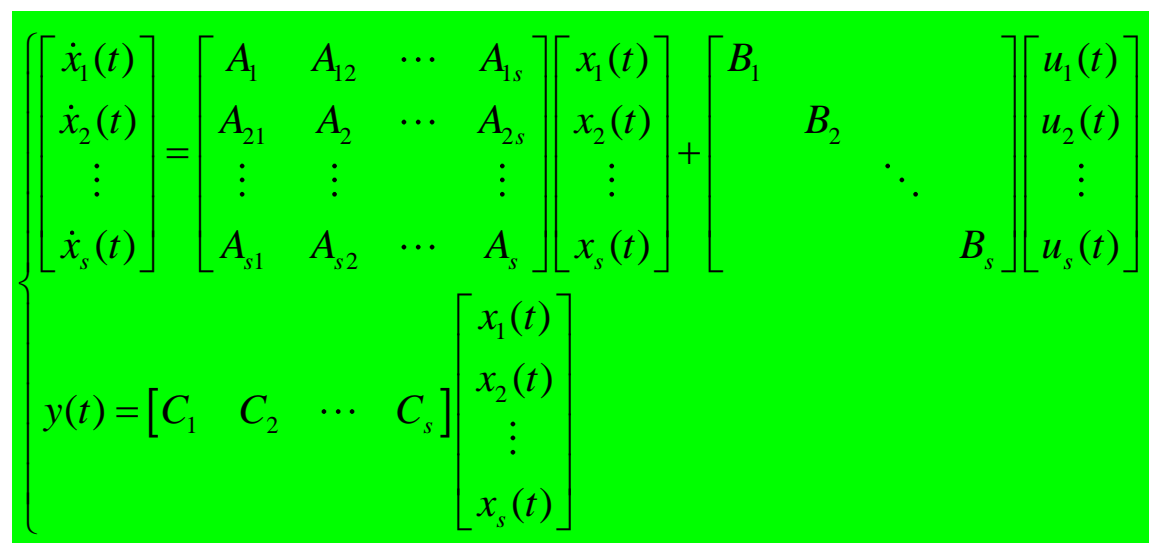

or

$$
\left\{\begin{array}{l}
\dot{x}_{i}(t)=A_{i} x_{i}(t)+\sum_{\substack{j=1 \\
j \neq i}}^{s} A_{i j} x_{j}(t)+B_{i} u_{i}(t) \\
y_{i}(t)=C_{i} x_{i}(t)
\end{array}, \quad i=1,2, \cdots, s\right.
$$

where $x_{i}(t) \in R^{n_{i}}, u_{i}(t) \in R^{m_{i}}$ and $y_{i}(t) \in R^{p}$ are state vector, input vector and output vector of the $i^{\text {th }}$ subsystem, respectively. Besides, $\sum_{i=1}^{s} n_{i}=n, \sum_{i=1}^{s} m_{i}=m$. $A_{i}, B_{i}$ and $C_{i}$ are constant matrices with appropriate dimension. $A_{i j}$ is an associated matrix between the $j^{\text {th }}$ subsystem and the $i^{\text {th }}$ subsystem $(i \neq j ; i, j=1,2, \cdots, s)$. The output of large-scale systems (1) is

$$
y(t)=\sum_{i=1}^{s} y_{i}(t)
$$

Let $r(t) \in R^{p}$ be the reference signal. Firstly, some fundamental assumptions are needed. 
A1. Suppose $\left(A_{i}, B_{i}\right)$ is stabilizable and matrix $\left[\begin{array}{cc}A_{i} & B_{i} \\ C_{i} & 0\end{array}\right]$ is of full row rank $(i=1,2, \cdots, s)$

A2. Suppose $\left(C_{i}, A_{i}\right)$ is observable $(i=1,2, \cdots, s)$;

A3. Suppose $r(t)$ is a piecewise-continuously differentiable function satisfying

$$
\lim _{t \rightarrow \infty} r(t)=r, \lim _{t \rightarrow \infty} \dot{r}(t)=0,
$$

where $r \in R^{p}$ is a constant vector. Moreover, $r(t)$ is previewable and the preview length is $l_{r}$. In the sense that the future values $r(\tau)\left(t \leq \tau \leq t+l_{r}\right)$ are available at each instant of time $t$ and suppose $r(\tau)=r\left(t+l_{r}\right)$ at the time $\tau>t+l_{r}$.

The tracking error is defined as follows:

$$
e(t)=y(t)-r(t)
$$

Our objective is to design a controller with preview compensation such that the output of systems (1) can track the reference signal asymptotically, i.e.,

$$
\lim _{t \rightarrow \infty} e(t)=\lim _{t \rightarrow \infty}[y(t)-r(t)]=0 .
$$

Owing to the fact that large-scale systems have such character with large scale, complex structure, numerous influencing factors and comprehensive functions, we need to solve a high-order algebraic Riccati equation. On the other hand, based on the formation of $y(t)$, the tracking error $e(t)$ can be written as following: 


$$
e(t)=\sum_{i=1}^{s} e_{i}(t)
$$

where

$$
e_{i}(t)=y_{i}(t)-\alpha_{i} r(t)
$$

with $\alpha_{i}$ satisfying $\alpha_{i}>0$ and $\sum_{i=1}^{s} \alpha_{i}=1$. Here, we consider each $\alpha_{i} r(t)$ as a virtual reference signal.

In fact, for arbitrary $i \in\{1,2, \cdots, s\}$, if

$$
\lim _{t \rightarrow \infty} e_{i}(t)=0
$$

then

$$
\lim _{t \rightarrow \infty} e(t)=\lim _{t \rightarrow \infty}\left(\sum_{i=1}^{s} e_{i}(t)\right)=\sum_{i=1}^{s}\left(\lim _{t \rightarrow \infty} e_{i}(t)\right)=0 .
$$

On the basis of above considerations, we decompose large-scale systems into several isolated subsystems based on decomposition-aggregation method, and design controller for each isolated subsystem to build up the controller of the large-scale systems. Regarding the influence of correlation on stability of the close-loop system, this paper will give sufficient conditions to ensure that the output of large-scale systems (1) asymptotically tracks the reference signal $r(t)$ by requiring that the total derivative of a positive definite Lyapunov function is negative. 
Briefly speaking, when the output $y_{i}(t)$ of each subsystem can track $\alpha_{i} r(t)$ without static error, the output $y(t)$ of system (1) can track the reference signal $r(t)$ accurately.

\section{Construction of the Augmented Large-scale Systems}

In what follows, for a given virtual reference signal $\alpha_{i} r(t)$, we will consider the optimal preview tracking problem of the following isolated subsystem

$$
\left\{\begin{array}{l}
\dot{x}_{i}(t)=A_{i} x_{i}(t)+B_{i} u_{i}(t) \\
y_{i}(t)=C_{i} x_{i}(t)
\end{array}\right.
$$

where tracking error is (2).

To obtain the optimal preview controller of system (3), we introduce following quadratic performance index

$$
J_{i}=\int_{0}^{\infty}\left[e_{i}^{\mathrm{T}}(t) Q_{e_{i}} e_{i}(t)+\dot{u}_{i}^{\mathrm{T}}(t) R_{i} \dot{u}_{i}(t)\right] d t,
$$

where $Q_{e_{i}}>0, R_{i}>0$.

Noting that corresponding performance index of system (3) contains the derivative of the input $u_{i}(t)$ rather than the input $u_{i}(t)$, we can introduce integral action into the final controller, which is good for eliminating steady-state error (Liao et al., 2003).

According to preview control theory, in order to design preview controller, it is 
desirable to construct an augmented system for each isolated subsystem. To avoid repeating, we construct the augmented system for systems (1) and let all associated matrices be zeros to gain the desired result.

For a given $i(i=1,2, \cdots, s)$, derivating both sides of (1) and (2) gives

$$
\begin{gathered}
\dot{e}_{i}(t)=\dot{y}_{i}(t)-\alpha_{i} \dot{r}(t)=C_{i} \dot{x}_{i}(t)-\alpha_{i} \dot{r}(t) . \\
\frac{d}{d t} \dot{x}_{i}(t)=A_{i} \dot{x}_{i}(t)+B_{i} \dot{u}_{i}(t)+\sum_{\substack{j=1 \\
j \neq i}}^{s} A_{i j} \dot{x}_{j}(t) .
\end{gathered}
$$

Letting $\quad z_{i}(\mathrm{t})=\left[\begin{array}{c}e_{i}(\mathrm{t}) \\ \dot{x}_{i}(\mathrm{t})\end{array}\right] \in R^{p+n_{i}}$ yields

$$
\left\{\begin{array}{l}
\dot{z}_{i}(t)=\tilde{A}_{i} z_{i}(t)+\tilde{B}_{i} \dot{u}_{i}(t)+\sum_{\substack{j=1 \\
j \neq i}}^{s} \tilde{A}_{i j} z_{j}(t)-\tilde{D}_{i} \dot{r}(t) \\
e_{i}(t)=\tilde{C}_{i}(t) z_{i}(t)
\end{array}\right.
$$

where

$$
\tilde{A}_{i}=\left[\begin{array}{cc}
0 & C_{i} \\
0 & A_{i}
\end{array}\right], \tilde{B}_{i}=\left[\begin{array}{c}
0 \\
B_{i}
\end{array}\right], \tilde{D}_{i}=\left[\begin{array}{c}
\alpha_{i} I_{p} \\
0
\end{array}\right], \tilde{\mathrm{C}}_{i}=\left[\begin{array}{ll}
I_{p} & 0
\end{array}\right], \tilde{A}_{i j}=\left[\begin{array}{cc}
0 & 0 \\
0 & A_{i j}
\end{array}\right] .
$$

They are $\left(p+n_{i}\right) \times\left(p+n_{i}\right), \quad\left(p+n_{i}\right) \times m_{i}, \quad\left(p+n_{i}\right) \times p, \quad p \times\left(p+n_{i}\right) \quad$ and

\section{$\left(p+n_{i}\right) \times\left(p+n_{i}\right)$ matrices, respectively.}

Write (7) as a compact form, i.e.,

$$
\dot{z}(t)=\tilde{A} z(t)+\tilde{B} \dot{u}(t)-\tilde{D} \dot{r}(t)
$$

where 


$$
\begin{gathered}
z(t)=\left[\begin{array}{c}
z_{1}(t) \\
z_{2}(t) \\
\vdots \\
z_{s}(t)
\end{array}\right], \quad \tilde{A}=\left[\begin{array}{cccc}
\tilde{A}_{1} & \tilde{A}_{12} & \cdots & \tilde{A}_{1 s} \\
\tilde{A}_{21} & \tilde{A}_{2} & \cdots & \tilde{A}_{2 s} \\
\vdots & \vdots & & \vdots \\
\tilde{A}_{s 1} & \tilde{A}_{s 2} & \cdots & \tilde{A}_{s}
\end{array}\right], \\
\dot{u}(t)=\left[\begin{array}{c}
\dot{u}_{1}(t) \\
\dot{u}_{2}(t) \\
\vdots \\
\dot{u}_{s}(t)
\end{array}\right], \quad \tilde{B}=\left[\begin{array}{cccc}
\tilde{B}_{1} & 0 & \cdots & 0 \\
0 & \tilde{B}_{2} & \cdots & 0 \\
\vdots & \vdots & & \vdots \\
0 & 0 & \cdots & \tilde{B}_{s}
\end{array}\right], \quad \tilde{D}=\left[\begin{array}{cccc}
\tilde{D}_{1} & 0 & \cdots & 0 \\
0 & \tilde{D}_{2} & \cdots & 0 \\
\vdots & \vdots & & \vdots \\
0 & 0 & \cdots & \tilde{D}_{s}
\end{array}\right] .
\end{gathered}
$$

Then system (8) is the desired augmented system.

Letting $\tilde{A}_{i j}=0(i \neq j ; i, j=1,2, \cdots, s)$, then the $i^{\text {th }}$ isolated augmented subsystem is

$$
\dot{z}_{i}(t)=\tilde{A}_{i} z_{i}(t)+\tilde{B}_{i} \dot{u}_{i}(t)-\tilde{D}_{i} \dot{r}(t)
$$

With the related variables of (9), the performance index in (4) is modified into the following form

$$
\tilde{J}_{i}=J_{i}+\int_{0}^{\infty}\left[\dot{x}_{i}^{\mathrm{T}}(t) Q_{x_{i}} \dot{x}_{i}(t)\right] d t=\int_{0}^{\infty}\left[z_{i}^{\mathrm{T}}(t) \tilde{Q}_{i} z_{i}(t)+\dot{u}_{i}^{\mathrm{T}}(t) R_{i} \dot{u}_{i}(t)\right] d t
$$

where $\tilde{Q}_{i}=\left[\begin{array}{cc}Q_{e_{i}} & 0 \\ 0 & Q_{x_{i}}\end{array}\right], Q_{x_{i}} \geq 0$.

\section{Main Results}

In this section, we will apply the standard result of preview control theorem to design the optimal preview controller for large-scale systems (1). Meanwhile, by means of Lyapunov stability theorem and the property of M-matrix, we also provide sufficient 
conditions to guarantee the asymptotic stability of the closed-loop system of system (8).

\subsection{Design of Optimal Preview Controllers for the Isolated Augmented}

\section{Subsystems}

The following lemma is obtained immediately by Liao et al. (2011).

Lemma 1: If $\left(\tilde{A}_{i}, \tilde{B}_{i}\right)$ is stabilizable and $\left(\tilde{Q}_{i}^{1 / 2}, \tilde{A}_{i}\right)$ is detectable, then the optimal control input of system (9) under performance index (10) is given by

$$
\dot{u}_{i}(t)=-R_{i}^{-1} \tilde{B}_{i}^{\mathrm{T}} P_{i} z_{i}(t)-R_{i}^{-1} \tilde{B}_{i}^{\mathrm{T}} g_{i}(t)
$$

where $P_{i} \in R^{\left(n_{i}+p\right) \times\left(n_{i}+p\right)}$ is a symmetric positive semi-definite solution to the algebraic Riccati equation

$$
\tilde{A}_{i}^{\mathrm{T}} P_{i}+P_{i} \tilde{A}_{i}-P_{i} \tilde{B}_{i} R_{i}^{-1} \tilde{B}_{i}^{\mathrm{T}} P_{i}+\tilde{Q}_{i}=0
$$

$g_{i}(t) \in R^{p}$ is the function satisfying

$$
g_{i}(t)=-\int_{0}^{l_{r}} \exp \left(\sigma \tilde{A}_{c i}^{\mathrm{T}}\right) P_{i} \tilde{D}_{i} \dot{r}(t+\sigma) d \sigma
$$

where

$$
\tilde{A}_{c i}=\tilde{A}_{i}-\tilde{B}_{i} R_{i}^{-1} \tilde{B}_{i}^{\mathrm{T}} P_{i}
$$

Remark 1: According to optimal control theory, when $\left(\tilde{Q}_{i}^{1 / 2}, \tilde{A}_{i}\right)$ is observable, there is a unique symmetric positive definite matrix solution to the algebraic Riccati equation (12). In the followings, the result will be used to show the closed-loop stability of the augmented large-scale system (8). 


\subsection{The Closed-loop Stability of the Augmented Large-scale Systems}

Based on the idea of decomposition-aggregation and the control inputs expressed by formula (11), we introduce a vector

$$
\dot{u}(t)=\left[\begin{array}{c}
\dot{u}_{1}(t) \\
\dot{u}_{2}(t) \\
\vdots \\
\dot{u}_{s}(t)
\end{array}\right]
$$

which happens to be the control input of system (8).

Substitute formulas (11) and (15) into system (8), then a closed-loop system is obtained

$$
\dot{z}(t)=\Phi z(t)+\theta(t)
$$

where $\Phi$ and $\theta(t)$ are

$$
\Phi=\left[\begin{array}{cccc}
\tilde{A}_{c 1} & \tilde{A}_{12} & \cdots & \tilde{A}_{1 s} \\
\tilde{A}_{21} & \tilde{A}_{c 2} & \cdots & \tilde{A}_{2 s} \\
\vdots & \vdots & & \vdots \\
\tilde{A}_{s 1} & \tilde{A}_{s 2} & \cdots & \tilde{A}_{c s}
\end{array}\right], \quad \theta(t)=\left[\begin{array}{c}
\theta_{1}(t) \\
\theta_{2}(t) \\
\vdots \\
\theta_{s}(t)
\end{array}\right] .
$$

Matrix $\tilde{A}_{c i}$, the diagonal elements of matrix $\Phi$, is given by formula (14). $\theta_{i}(t)$ is $\theta_{i}(t)=-\tilde{B}_{i} R_{i}^{-1} \tilde{B}_{i}^{\mathrm{T}} g_{i}(t)-\tilde{D}_{i} \dot{r}(t)$ and $g_{i}(t)$ is given by formula (13).

Upon the foundation of Lemma 1, the sufficient conditions for asymptotic stability of system (16) are obtained.

Theorem 1: Suppose 
(1) $\left(\tilde{A}_{i}, \tilde{B}_{i}\right)$ is stabilizable and $\left(\tilde{Q}_{i}^{1 / 2}, \tilde{A}_{i}\right)$ is observable;

(2) inequality $\left\|P_{i} \tilde{A}_{i j}\right\| \leq \frac{l_{i j}}{2}(i \neq j ; i, j=1,2, \cdots, s)$ holds;

(3) matrix

$$
D=\left[\begin{array}{cccc}
-\lambda_{1} & l_{12} & \cdots & l_{1 s} \\
l_{21} & -\lambda_{2} & \cdots & l_{2 s} \\
\vdots & \vdots & & \vdots \\
l_{s 1} & l_{s 2} & \cdots & -\lambda_{s}
\end{array}\right]
$$

is stable, then the zero solution of system (16) is asymptotically stable, where $\lambda_{i}$ is given by $-\lambda_{i}=\lambda_{\max }\left[-\tilde{Q}_{i}-P_{i} \tilde{B}_{i} R_{i}^{-1} \tilde{B}_{i}^{\mathrm{T}} P_{i}\right](i=1,2, \cdots, s)$.

Proof. the proof can be found in Appendix.

Now the asymptotic stability of system (16) implies

$$
\lim _{t \rightarrow \infty} e_{i}(t)=0,
$$

thus

$$
\lim _{t \rightarrow \infty} e(t)=0
$$

Namely, under the conditions given by Theorem 2, system (1) can track the reference signal $r(t)$ without static error.

Remark 2: If $Q_{x_{i}}$ is further required to be positive definite in the performance index function (10), then $\tilde{Q}_{i}$ is also positive definite, and as a direct result, $\left(\tilde{Q}_{i}^{1 / 2}, \tilde{A}_{i}\right)$ is observable obviously. Due to $\lambda_{\max }\left[-\tilde{Q}_{i}-P_{i} \tilde{B}_{i} R_{i}^{-1} \tilde{B}_{i}^{\mathrm{T}} P_{i}\right] \leq \lambda_{\max }\left(-\tilde{Q}_{i}\right)$, a 
straightforward consequence of Theorem 1 is that

\section{Corollary 1: Suppose}

(1) $\left(\tilde{A}_{i}, \tilde{B}_{i}\right)$ is stabilizable and $\tilde{Q}_{i}>0$;

(2) inequality $\left\|P_{i} \tilde{A}_{i j}\right\| \leq \frac{l_{i j}}{2}(i=1,2, \cdots, s)$ holds;

(3) matrix $D$ is stable in (17),

then the zero solution of system (16) is asymptotically stable where $\lambda_{i}=\lambda_{\text {min }}\left(\tilde{Q}_{i}\right)$ $(i=1,2, \cdots, s)$.

\subsection{Discussion of Stabilizability and Observability}

In what follows, we need to discuss the conditions that can guarantee the stabilizability (controllability) of $\left(\tilde{A}_{i}, \tilde{B}_{i}\right)$ and the detectability (observability) of $\left(\tilde{Q}_{i}^{1 / 2}, \tilde{A}_{i}\right)$.

Theorem 2: The sufficient and necessary conditions for the stabilizability (controllability) of $\left(\tilde{A}_{i}, \tilde{B}_{i}\right)$ are that $\left(A_{i}, B_{i}\right)$ is stabilizable (controllable) and matrix $\left[\begin{array}{cc}A_{i} & B_{i} \\ C_{i} & 0\end{array}\right]$ is of full row $\operatorname{rank}(i=1,2, \cdots, s)$.

Proof. Noting the structure of matrices $\tilde{A}_{i}$ and $\tilde{B}_{i}$, the theorem is a direct conclusion of [5].

Theorem 3: If $Q_{e_{i}}>0$, then the sufficient and necessary condition for the 
observability of matrix $\left(\tilde{Q}_{i}^{1 / 2}, \tilde{A}_{i}\right)$ is that matrix $\left[\begin{array}{c}A_{i}-\lambda I \\ C_{i} \\ Q_{x_{i}}^{1 / 2}\end{array}\right]$ is of full column rank for all complex $\lambda$.

Proof. The Popov-Belevitch-Hautus ( $\mathrm{PBH}$ ) rank test is used to prove the theorem [11]. Noting that

$$
\left[\begin{array}{c}
\tilde{A}_{i}-\lambda I \\
\tilde{Q}_{i}^{1 / 2}
\end{array}\right]=\left[\begin{array}{cc}
-\lambda I & C_{i} \\
0 & A_{i}-\lambda I \\
\hdashline Q_{e_{i}}^{1 / 2} & 0 \\
0 & Q_{x_{i}}^{1 / 2}
\end{array}\right]
$$

Because of $Q_{e_{i}}>0$, we take elementary transformation and get

$$
\left[\begin{array}{c}
\tilde{A}_{i}-\lambda I \\
\tilde{Q}_{i}^{1 / 2}
\end{array}\right] \rightarrow\left[\begin{array}{cc}
0 & C_{i} \\
0 & A_{i}-\lambda I \\
\hdashline I & 0 \\
0 & Q_{x_{i}}^{1 / 2}
\end{array}\right] \rightarrow\left[\begin{array}{cc}
I & 0 \\
0 & A_{i}-\lambda I \\
0 & C_{i} \\
0 & Q_{x_{i}}^{1 / 2}
\end{array}\right]
$$

It is well known that elementary transformation does not change the rank of a matrix. Therefore, for any complex $\lambda,\left[\begin{array}{c}\tilde{A}_{i}-\lambda I \\ \tilde{Q}_{i}^{1 / 2}\end{array}\right]$ has full column rank if and only if $\left[\begin{array}{c}A_{i}-\lambda I \\ C_{i} \\ Q_{x_{i}}^{1 / 2}\end{array}\right]$ has full column rank, which is exactly the conclusion of Theorem 3 based on PBH rank test.

Two corollaries are obtained as follows. 
Corollary 2: Letting $Q_{e_{i}}>0$. If $\left(\tilde{C}_{i}, \tilde{A}_{i}\right)$ is observable, then $\left(\tilde{Q}_{i}^{1 / 2}, \tilde{A}_{i}\right)$ is observable. In particular, if also $Q_{x_{i}}=0$ under the foundation of $Q_{e_{i}}>0$, then the sufficient and necessary condition for the observability of matrix $\left(\tilde{Q}_{i}^{1 / 2}, \tilde{A}_{i}\right)$ is that $\left(\tilde{C}_{i}, \tilde{A}_{i}\right)$ is observable.

Corollary 3: If $Q_{e_{i}}>0$ and $Q_{x_{i}}>0$, then $\left(\tilde{Q}_{i}^{1 / 2}, \tilde{A}_{i}\right)$ is observable.

\subsection{The Preview Controller of the Large-scale Systems}

When the conditions of Theorem 1 hold, $u(t)$ is derived from formula (15) and the controller of systems (1) is gained as well. Therefore, we get the main theorem in this paper.

\section{Theorem 4: Suppose}

(1) A1-A3 hold;

(2) $Q_{e_{i}}>0, R_{i}>0(i=1,2, \cdots, s)$;

(3) $\left\|P_{i} \tilde{A}_{i j}\right\| \leq \frac{l_{i j}}{2}(i \neq j ; i, j=1,2, \cdots, s)$;

(4) matrix $D$ is stable in (17),

and let $x_{i}(t)=0, \quad u_{i}(t)=0, \quad(i=1,2, \cdots, s), \quad r(t)=0$ for $t<0$, then the optimal preview tracking of large-scale systems (1) is achieved by the following controller

$$
u(t)=\left[\begin{array}{llll}
u_{1}^{T}(t) & u_{2}^{T}(t) & \cdots & u_{s}^{T}(t)
\end{array}\right]^{T}
$$


with

$$
u_{i}(t)=-K_{e_{i}} \int_{0}^{t} e_{i}(\sigma) d \sigma-K_{x_{i}} x_{i}(t)+f_{i}(t)
$$

where $K_{e_{i}}=R_{i}^{-1} \tilde{B}_{i}^{\mathrm{T}} P_{i e_{i}}, K_{x_{i}}=R_{i}^{-1} \tilde{B}_{i}^{\mathrm{T}} P_{i x_{i}}$ and $P_{i}=\left[\begin{array}{ll}P_{i e_{i}} & P_{i x_{i}}\end{array}\right]$, and the expression of $f_{i}(t) \in R^{p}$ is given by $f_{i}(t)=R_{i}^{-1} \tilde{B}_{i}^{\mathrm{T}} \int_{0}^{l_{r}} \exp \left(\sigma \tilde{A}_{c i}^{\mathrm{T}}\right) P_{i} \tilde{D}_{i} r(t+\sigma) d \sigma$. In matrix $D$, $\lambda_{i}=\lambda_{\max }\left[\tilde{Q}_{i}+P_{i} \tilde{B}_{i} R_{i}^{-1} \tilde{B}_{i}^{\mathrm{T}} P_{i}\right](i=1,2, \cdots, s)$.

Proof. It follows from conditions (1) - (4) that Theorem 1 holds, namely, the zero solution of closed-loop system (16) is asymptotically stable. Therefore, as a component of $z_{i}(t), e_{i}(t)$ will tend to zero as time goes to infinity and so will $e(t)$ because of $e(t)=\sum_{i=1}^{s} e_{i}(t)$. That is to say, the output of large-scale systems (1) achieves optimal tracking of reference signal $r(t)$ under controller (11). In order to derive $u_{i}(t)$ from controller (11), we select an $L$ satisfying $L \geq l_{r}$. Then, integrating (11) on interval $[-L, t)$ yields controller (19).

Remark 3: As mentioned by Corollary 1 , if $\tilde{Q}_{i}>0$, then $\lambda_{i}$ of Theorem 4 can be change to $\lambda_{\min }\left(\tilde{Q}_{i}\right)$.

\section{Numerical Simulation}

Example 1. Consider large-scale systems containing two subsystems 


$$
\left\{\begin{array}{l}
\dot{x}_{i}(t)=A_{i} x_{i}(t)+\sum_{\substack{j=1 \\
j \neq i}}^{2} A_{i j} x_{j}(t)+B_{i} u_{i}(t) \\
y_{i}(t)=C_{i} x_{i}(t)
\end{array} \quad i=1,2\right.
$$

and its coefficient matrixcs are respectively

$$
\begin{aligned}
& A_{1}=\left[\begin{array}{ccc}
-3 & 0 & 1 \\
0 & -1 & 1 \\
2 & 0 & -2
\end{array}\right], A_{12}=\left[\begin{array}{cc}
0 & 0.0001 \\
0.0001 & -0.0001 \\
0.0001 & 0.0002
\end{array}\right], B_{1}=\left[\begin{array}{c}
1 \\
-1 \\
-1
\end{array}\right], C_{1}=\left[\begin{array}{lll}
1 & 1 & 0
\end{array}\right], \\
& A_{2}=\left[\begin{array}{cc}
-1 & 0 \\
1 & -2
\end{array}\right], A_{21}=\left[\begin{array}{ccc}
0.0001 & 0.0002 & 0 \\
0 & -0.0001 & 0.0001
\end{array}\right], B_{2}=\left[\begin{array}{l}
1 \\
1
\end{array}\right], C_{2}=\left[\begin{array}{ll}
0 & 1
\end{array}\right] .
\end{aligned}
$$

By verifying, $\left(A_{i}, B_{i}\right)$ is stabilizable, matrix $\left[\begin{array}{cc}A_{i} & B_{i} \\ C_{i} & 0\end{array}\right]$ is of full row rank and $\left(C_{i}, A_{i}\right)$ is observable $(i=1,2)$. Thus, the system satisfies the basic assumptions in this paper.

Let the reference signal be

$$
r(t)=\left\{\begin{array}{lc}
0, & t \leq 8 \\
t-8, & 8<t \leq 9 \\
1, & t>9
\end{array}\right.
$$

Moreover, put the weight matrices in performance index function (10) be

$$
\tilde{Q}_{1}=\left[\begin{array}{c:ccc}
50 & 0 & 0 & 0 \\
\hdashline 0 & 0.005 & 0 & 0 \\
0 & 0 & 0.005 & 0 \\
0 & 0 & 0 & 0
\end{array}\right], R_{1}=0.1, \quad \tilde{Q}_{2}=\left[\begin{array}{c:cc}
50 & 0 & 0 \\
\hdashline 0 & 0.005 & 0 \\
0 & 0 & 0
\end{array}\right], R_{2}=0.1 .
$$

Then solving algebraic Riccati equations (12) by Matlab, we get the following 
positive definite solutions

$$
P_{1}=\left[\begin{array}{cccc}
23.9120 & 4.0678 & 5.1047 & 1.1991 \\
4.0678 & 0.9338 & 1.2041 & 0.3605 \\
5.1047 & 1.2041 & 1.5701 & 0.4751 \\
1.1991 & 0.3605 & 0.4751 & 0.1883
\end{array}\right], \quad P_{2}=\left[\begin{array}{ccc}
15.1280 & 0.1654 & 2.0707 \\
0.1654 & 0.0257 & 0.0595 \\
2.0707 & 0.0595 & 0.4318
\end{array}\right],
$$

and the gain matrices in controller (19) are

$$
\begin{gathered}
K_{e_{1}}=-22.3607, K_{x_{1}}=\left[\begin{array}{lll}
-6.3088 & -8.4108 & -3.0295
\end{array}\right], \\
K_{e_{2}}=22.3607, K_{x_{2}}=\left[\begin{array}{ll}
0.8521 & 4.9134
\end{array}\right] .
\end{gathered}
$$

Let initial value be $x_{1}(0)=\left[\begin{array}{lll}0 & 0 & 0\end{array}\right]^{T}, x_{2}(0)=\left[\begin{array}{ll}0 & 0\end{array}\right]^{T}$, and set $\alpha_{1}=0.1, \alpha_{2}=0.9$ in formula (2). We perform simulations for three situations, that is, the preview lengths of the reference signals are $l_{r}=0(s), l_{r}=0.01(s), l_{r}=0.02(s)$, respectively. Figure 1 shows the output response of systems (1) under control input (19). Figures 2-3 illustrate the output responses of subsystems 1 and 2, respectively. Figures 4-5 plot the optimal control inputs of subsystems 1 and 2, respectively. 


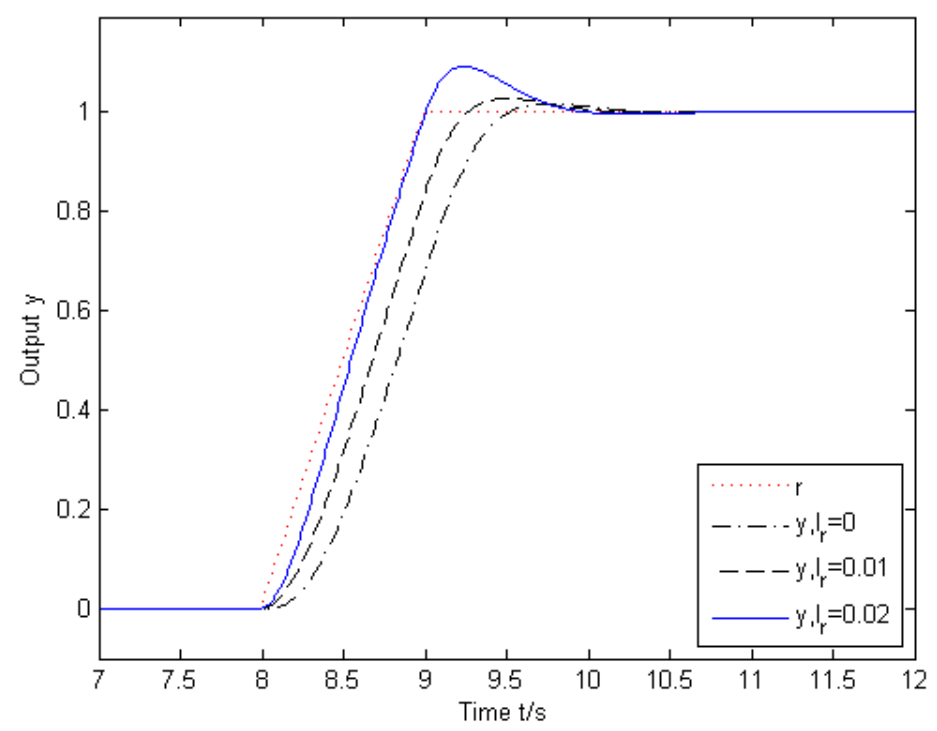

Figure 1. The output responses of the large-scale systems for different preview lengths

It is observed from Figure 1 that the output of large-scale systems (1) can track the reference signal quickly with preview compensation. Within certain range, the longer the preview length is, the better the effect of tracking. 


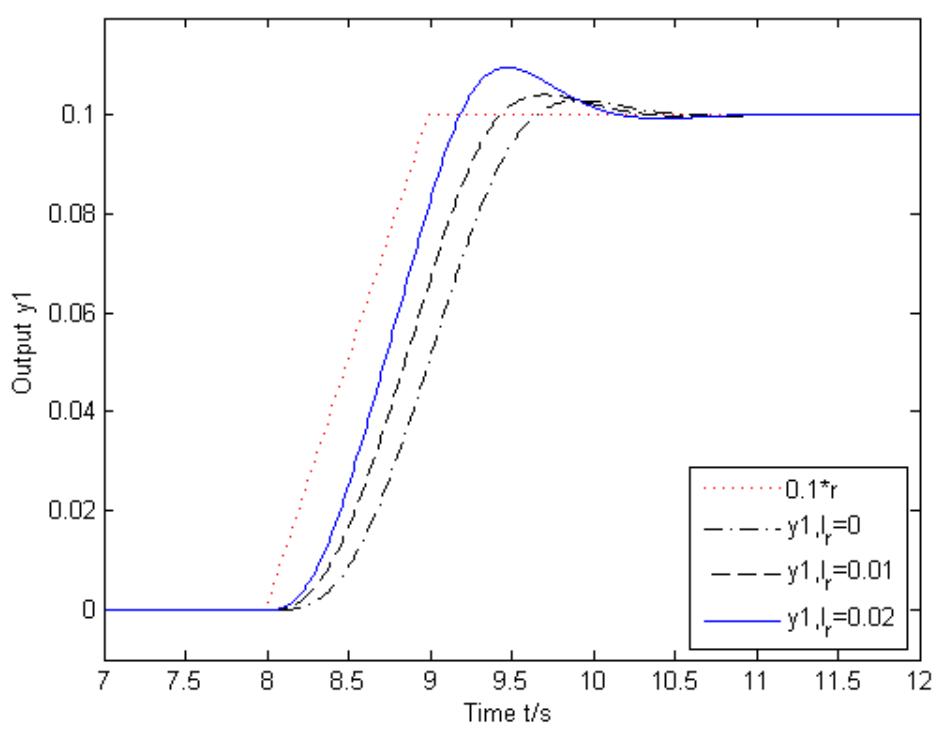

Figure 2. The output responses of subsystem 1 for different preview lengths

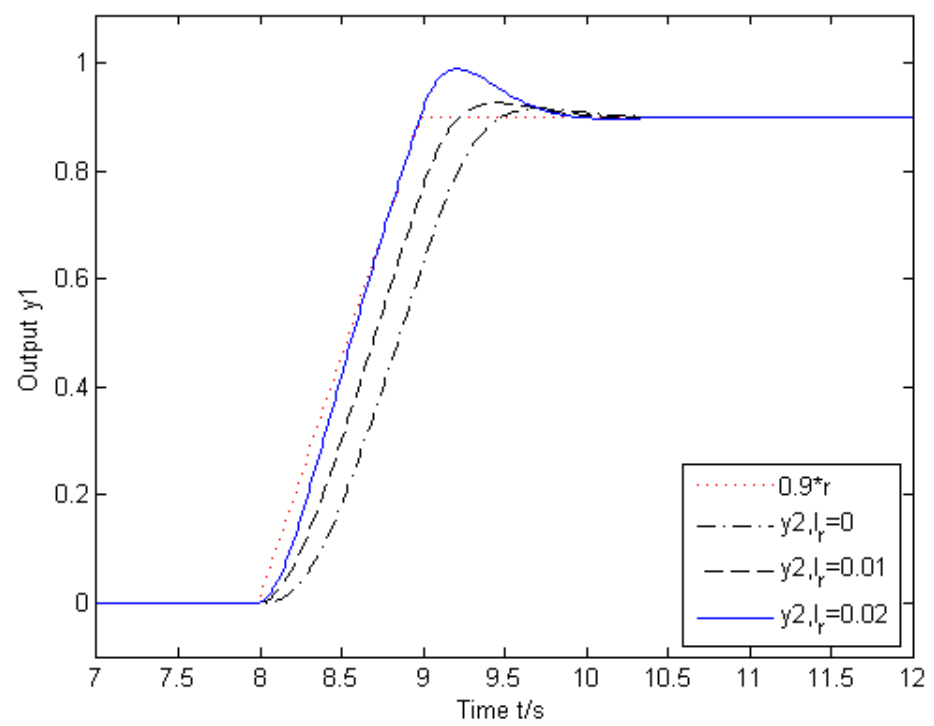

Figure 3. The output responses of subsystem 2 for different preview lengths 


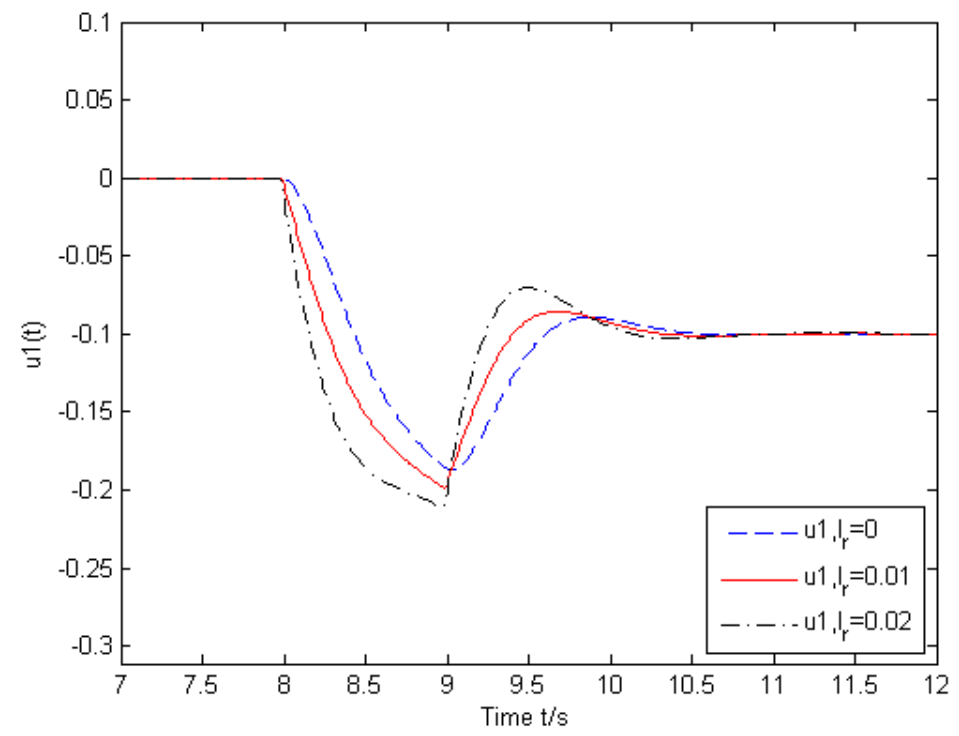

Figure 4. The optimal control input of subsystem 1 under different preview lengths

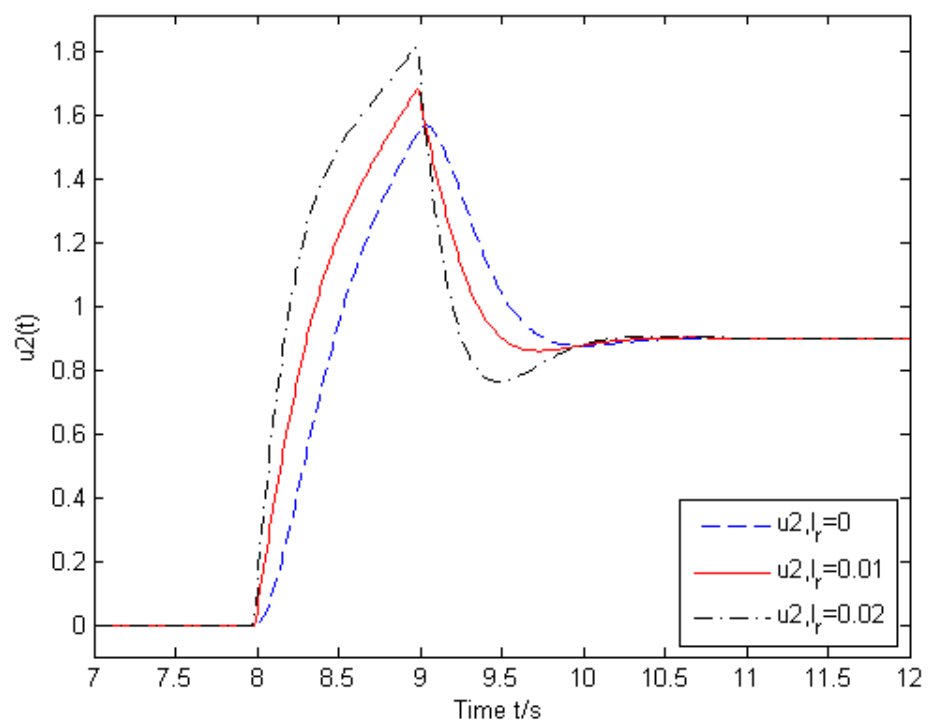

Figure 5. The optimal control input of subsystem 2 under different preview lengths

From Figures 2 and 3, it can be found that the designed controller can make the 
output of subsystem track the given virtual reference signal asymptotically. As a result, the tracking errors $e_{1}(t)$ and $e_{2}(t)$ will tend to zero as time goes to infinity.

Combining with Figure 1 and formula (2), it indirectly proves the validity of the reduced-order design method proposed in this paper. Moreover, we can also find that the controller has the ability to accelerate the tracking speed. In addition, although the overshoot rises up gradually with moderate increase of the preview length, the tracking performance is improved and the settling time decreases as well.

Example 2. To further illustrate the efficiency of the designed controller. We consider the optimal control problem that a large-scale system tracks a previewable step reference signal. The dynamics of the subsystems are described as follows

$$
\left\{\begin{array}{l}
\dot{x}_{i}(t)=A_{i} x_{i}(t)+\sum_{\substack{j=1 \\
j \neq i}}^{2} A_{i j} x_{j}(t)+B_{i} u_{i}(t) \\
y_{i}(t)=C_{i} x_{i}(t)
\end{array}, \quad i=1,2\right.
$$

and the corresponding coefficient matrices are

$$
\begin{aligned}
& A_{1}=\left[\begin{array}{rrr}
-1 & 1 & -1 \\
0 & -1 & -1 \\
1 & 0 & -0.5
\end{array}\right], A_{12}=\left[\begin{array}{rrrr}
0 & 0 & 0.01 & 0.01 \\
0.01 & -0.01 & 0 & 0 \\
0.01 & 0.02 & 0 & 0
\end{array}\right], B_{1}=\left[\begin{array}{l}
1 \\
1 \\
0
\end{array}\right], C_{1}=\left[\begin{array}{lll}
0 & 0 & 0
\end{array}\right], \\
& A_{2}=\left[\begin{array}{rrrr}
-3 & 1 & 0 & 0 \\
2 & -2 & 0 & 0 \\
0 & 0 & -1 & 2 \\
-3 & -5 & 0 & -2
\end{array}\right], A_{21}=\left[\begin{array}{rrr}
0.01 & 0.02 & 0 \\
0 & -0.01 & 0.01 \\
-0.01 & 0 & 0 \\
0 & 0.01 & 0.02
\end{array}\right], B_{2}=\left[\begin{array}{r}
1 \\
-1.6 \\
1 \\
0
\end{array}\right], C_{2}=\left[\begin{array}{llll}
1 & -1 & 1 & 1.5
\end{array}\right] .
\end{aligned}
$$

Assume that the step reference signal has the following form 


$$
r(t)= \begin{cases}0, & t \leq 8 \\ 1, & t>8\end{cases}
$$

and satisfies the assumption of preview in A1. That is to say, the value of $r(\tau)$ is available to large-scale systems (19) in interval $\left[t, t+l_{r}\right]$ at time $t$.

Because of $C_{1}=\left[\begin{array}{lll}0 & 0 & 0\end{array}\right]$, the output of large-scale systems (19) is $y(t)=y_{2}(t)$. Therefore, the optimal preview tracking problem of large-scale systems (19) can be decomposed into an optimal regulation problem of subsystem 1 and an optimal preview tracking problem of subsystem 2 .

Select the weight matrices associated with the performance index function (10) as

$$
Q_{x_{1}}=\left[\begin{array}{ccc}
0.4 & 0 & 0 \\
0 & 0.4 & 0 \\
0 & 0 & 0.4
\end{array}\right], R_{1}=0.4, \tilde{Q}_{2}=\left[\begin{array}{c:c}
Q_{e_{2}} & 0 \\
\hdashline 0 & Q_{x_{2}}
\end{array}\right]=\left[\begin{array}{c:cccc}
60 & 0 & 0 & 0 & 0 \\
\hdashline 0 & 15 & 0 & 0 & 0 \\
0 & 0 & 3 & 0 & 0 \\
0 & 0 & 0 & 9 & 0 \\
0 & 0 & 0 & 0 & 6
\end{array}\right], R_{2}=0.3
$$

By applying $\mathrm{PBH}$ rank test, it can be calculated that $\left(A_{i}, B_{i}\right)$ is stabilizable, $i=1,2, \quad\left(C_{2}, A_{2}\right)$ is observable. Moreover, routine calculation gives that matrix $\left[\begin{array}{cc}A_{2} & B_{2} \\ C_{2} & 0\end{array}\right]$ is of full row rank. Noting that $Q_{x_{1}}$ and $Q_{e_{2}}$ are positive definite matrices. Then, according to the conclusions of Lemma1, Theorem 2, and Corollaries 2-3, we know that the following algebraic Riccati equations have positive definite solutions:

$$
A_{1}^{\mathrm{T}} P_{1}+P_{1} A_{1}-P_{1} B_{1} R_{1}^{-1} B_{1}^{\mathrm{T}} P_{1}+Q_{x_{1}}=0
$$




$$
\tilde{A}_{2}^{\mathrm{T}} P_{2}+P_{2} \tilde{A}_{2}-P_{2} \tilde{B}_{2} R_{2}^{-1} \tilde{B}_{2}^{\mathrm{T}} P_{2}+\tilde{Q}_{2}=0
$$

Solving the above equations by Matlab gives

$$
\begin{gathered}
P_{1}=\left[\begin{array}{rrrr}
0.21764 & 0.01695 & 0.08644 \\
0.01695 & 0.17222 & -0.07279 \\
0.08644 & -0.07279 & 0.37225
\end{array}\right], \\
P_{2}=\left[\begin{array}{rrrrr}
16.98680 & 1.25749 & -1.74038 & 0.20054 & 2.32069 \\
1.25749 & 6.39163 & 4.04398 & 0.27471 & -0.79782 \\
-1.74038 & 4.04398 & 4.14416 & 0.90008 & -1.14899 \\
0.20054 & 0.27471 & 0.90008 & 2.35239 & 0.46133 \\
2.32069 & -0.79782 & -1.14899 & 0.46133 & 1.82212
\end{array}\right],
\end{gathered}
$$

the control gain matrices in (18) are

$$
\begin{gathered}
K_{x_{1}}=\left[\begin{array}{lll}
0.58649 & 0.47293 & 0.03411
\end{array}\right], \\
K_{e_{2}}=[14.14214], K_{x_{2}}=\left[\begin{array}{llll}
0.65325 & -5.62197 & 3.95656 & 5.00629
\end{array}\right] .
\end{gathered}
$$

We take $l_{i j}=2\left\|P_{i} A_{i j}\right\|_{2}$, then

$$
\begin{aligned}
& l_{12}=2\left\|P_{1} A_{12}\right\|_{2}=0.0189 \\
& l_{21}=2\left\|P_{2} A_{21}\right\|_{2}=0.2620
\end{aligned}
$$

In addition,

$$
\begin{aligned}
& -\lambda_{1}=\lambda_{\max }\left(-Q_{x_{1}}-P_{1} B_{1} R_{1}^{-1} B_{1}^{T} P_{1}\right)=-0.4000 \\
& -\lambda_{2}=\lambda_{\max }\left(-\tilde{Q}_{2}-P_{2} \tilde{B}_{2} R_{2}^{-1} \tilde{B}_{2}^{T} P_{2}\right)=-3.5864
\end{aligned}
$$

By calculation, the eigenvalues of $D=\left[\begin{array}{rr}-0.4000 & 0.0189 \\ 0.2620 & -3.5864\end{array}\right]$ are -0.3984 and 
-3.5880 , which indicate that $D$ is stable. That is to say, the conditions (3) and (4) of Theorem 4 hold. Hence, the optimal preview tracking of large-scale systems (19) can be achieved by controller (18).

Let initial value be $x_{1}(0)=\left[\begin{array}{lll}0 & 0 & 0\end{array}\right]^{T}, x_{2}(0)=\left[\begin{array}{llll}0 & 0 & 0 & 0\end{array}\right]^{T}$. The simulation will be carried out for three situations, that is, the preview lengths of the reference signal are $l_{r}=0(s), l_{r}=0.08(s), l_{r}=0.15(s)$, respectively.

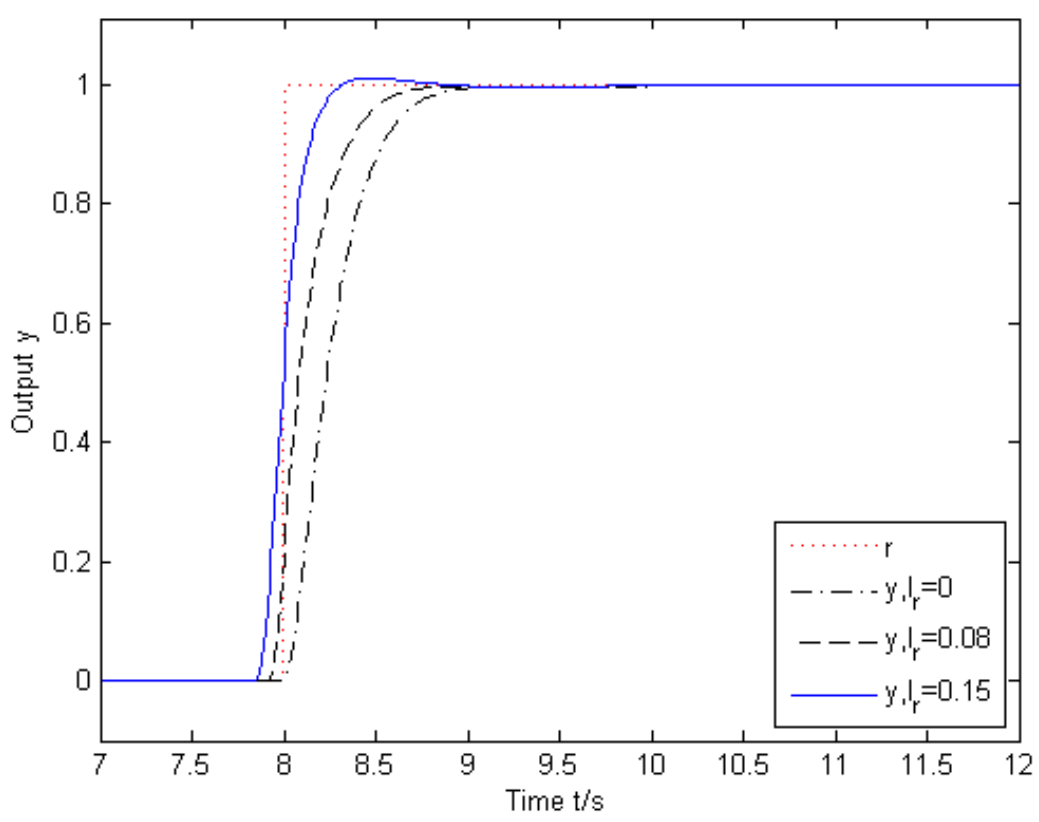

Figure 6. The output responses of the large-scale systems for different preview lengths 


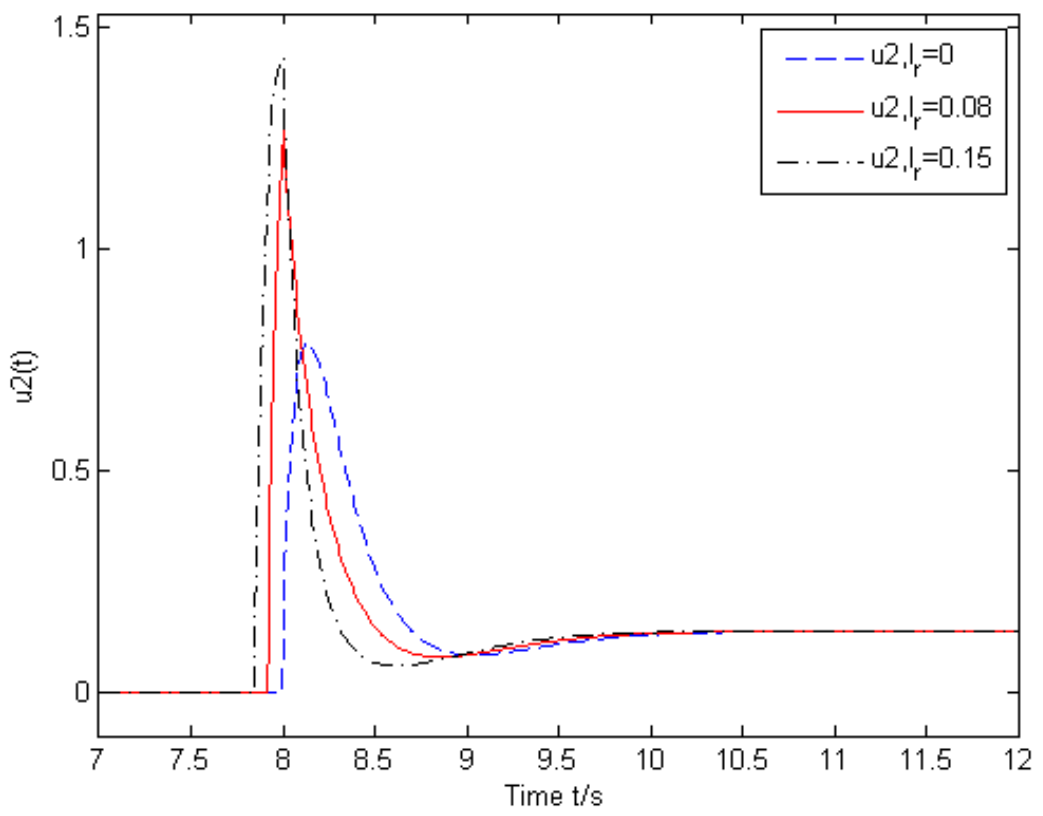

Figure 7. The optimal control input of subsystem 2 under different preview lengths

Figure 6 shows the output responses of large-scale systems (19) under control input (18). Figure 7 shows the optimal control input of subsystem 2. It is seen from Figure 6 that $y(t)$ can track the reference signal accurately whether preview information exists or not. Furthermore, compared with the case that $l_{r}=0(s)$, it can be also observed from figures 6 and 7 that the controller with preview compensation can improve the dynamic performance effectively, such as accelerating transient response, reducing tracking error.

Remark 4: In Example 1, although $\tilde{Q}_{i}$ is a positive semi-definite matrix, the 
observability of the matrix $\left(C_{i}, A_{i}\right)$ can ensure that $\left(\tilde{Q}_{i}^{1 / 2}, \tilde{A}_{i}\right)$ is observable. And $\lambda_{i}$ should be $\lambda_{i}=\lambda_{\max }\left[\tilde{Q}_{i}+P_{i} \tilde{B}_{i} R_{i}^{-1} \tilde{B}_{i}^{\mathrm{T}} P_{i}\right]$.

\section{Conclusion}

The optimal preview control problem for a class of linear continuous-time large-scale systems has been settled in this paper. Owing to the integral in the expression of control input (11), this paper has not constructed the Lyapunov function for the closed-loop large-scale systems directly, but for its corresponding homogeneous linear systems. Based on the stable conditions of the homogeneous linear systems and assumption A3, the sufficient conditions have been given to guarantee the global asymptotic stability of the closed-loop large-scale systems. The simulation example has illustrated the effectiveness of the designed controller. How to extend the current conclusions to the discrete-time large-scale systems setting is a future topic worthy of further investigation.

\section{Conflict of Interests}

The authors declare that there is no conflict of interest.

\section{Funding}


This work was supported by the National Natural Science Foundation of China [grant number 61174209].

\section{Appendix (Proof of Theorem 1)}

Proof. We first prove the asymptotic stability of the zero solution of system

$$
\dot{z}(t)=\Phi z(t)
$$

which is the homogenous linear system of system (16). When assumptions 1-3 hold, it follows from optimal control theory that the solution $P_{i}$ of algebraic Riccati equation is positive definite if $\left(\tilde{Q}_{i}^{1 / 2}, \tilde{A}_{i}\right)$ is observable. Using $P_{i}$ to construct a function $V_{i}\left(z_{i}\right)=z_{i}^{\mathrm{T}} P_{i} z_{i}$, then $V_{i}$ is a positive definite quadratic form with respect to $z_{i}$. Taking the total derivative of function $V_{i}$ regarding $t$ along the trajectory of system (18) gives

$$
\left.\dot{V}_{i}\right|_{(18)}=\dot{z}_{i}^{\mathrm{T}} P_{i} z_{i}+z_{i}^{\mathrm{T}} P_{i} \dot{z}_{i}=z_{i}^{\mathrm{T}}\left(P_{i} \tilde{A}_{c i}+\tilde{A}_{c i}^{T} P_{i}\right) z_{i}+2 \sum_{\substack{j=1 \\ j \neq i}}^{s} z_{i}^{\mathrm{T}} P_{i} A_{i j} z_{j} .
$$

According to the expression of matrix $\tilde{A}_{c i}$, we get

$$
P_{i} \tilde{A}_{c i}+\tilde{A}_{c i}^{T} P_{i}=\left(P_{i} \tilde{A}_{i}+\tilde{A}_{i}^{\mathrm{T}} P_{i}-P_{i} \tilde{B}_{i} R_{i}^{-1} \tilde{B}_{i}^{\mathrm{T}} P_{i}\right)-P_{i} \tilde{B}_{i} R_{i}^{-1} \tilde{B}_{i}^{\mathrm{T}} P_{i}
$$

Because $P_{i}$ is the solution of algebraic Riccati equation (12), the formula $P_{i} \tilde{A}_{i}+\tilde{A}_{i}^{\mathrm{T}} P_{i}-P_{i} \tilde{B}_{i} R_{i}^{-1} \tilde{B}_{i}^{\mathrm{T}} P_{i}=-\tilde{Q}_{i}$ holds. Hence

$$
\lambda_{\max }\left(P_{i} \tilde{A}_{c i}+\tilde{A}_{c i}^{T} P_{i}\right)=\lambda_{\max }\left[-\tilde{Q}_{i}-P_{i} \tilde{B}_{i} R_{i}^{-1} \tilde{B}_{i}^{\mathrm{T}} P_{i}\right]=-\lambda_{i},
$$


and thereby an estimation of $\left.\dot{V}_{i}\right|_{(18)}$ is

$$
\left.\dot{V}_{i}\right|_{(18)} \leq-\lambda_{i}\left\|z_{i}\right\|^{2}+2 \sum_{\substack{j=1 \\ j \neq i}}^{s}\left\|P_{i} \tilde{A}_{i j}\right\|\left\|z_{i}\right\|\left\|z_{j}\right\| \leq\left\|z_{i}\right\|\left(-\lambda_{i}\left\|z_{i}\right\|+\sum_{\substack{j=1 \\ j \neq i}}^{s} l_{i j}\left\|z_{j}\right\|\right), \quad(i=1,2, \cdots, s) .
$$

Furthermore,

$$
\left[\begin{array}{c}
\left.\dot{V}_{1}\right|_{(18)} \\
\left.\dot{V_{2}}\right|_{(18)} \\
\vdots \\
\left.\dot{V_{s}}\right|_{(18)}
\end{array}\right] \leq\left[\begin{array}{llll}
\left\|z_{1}\right\| & & & \\
& \left\|z_{2}\right\| & & \\
& & \ddots & \\
& & & \left\|z_{s}\right\|
\end{array}\right] D\left[\begin{array}{c}
\left\|z_{1}\right\| \\
\left\|z_{2}\right\| \\
\vdots \\
\left\|z_{s}\right\|
\end{array}\right] .
$$

Now we turn to the key part of the proof. Noting that matrix $D$ is a stable Metzler matrix, thus there exists a diagonal matrix $K=\operatorname{diag}\left(k_{1}, k_{2}, \cdots, k_{s}\right)$, where $k_{i}>0$ holds for any $i=1,2, \cdots, s$, such that matrix $K D+D^{\mathrm{T}} K$ is negative definite (Liao, 1985). Using the elements of matrix $K$, we construct a quadratic function

$$
V=\sum_{i=1}^{s} k_{i} V_{i}=\left(k_{1}, k_{2}, \cdots, k_{s}\right)\left(\begin{array}{c}
V_{1} \\
V_{2} \\
\vdots \\
V_{s}
\end{array}\right),
$$

then $V$ is a positive definite quadratic form relating to vector $\left[\begin{array}{llll}z_{1}^{T} & z_{2}^{T} & \cdots & z_{s}^{T}\end{array}\right]^{T}$. Consider $V$ as a Lyapunov function of system (18), clearly, it is positive definite. Moreover, it has an infinitesimal upper bound as well as a radially unbounded property. Calculating the time derivative of $V$ along the trajectory of system (18) 
and estimating its upper bound yields

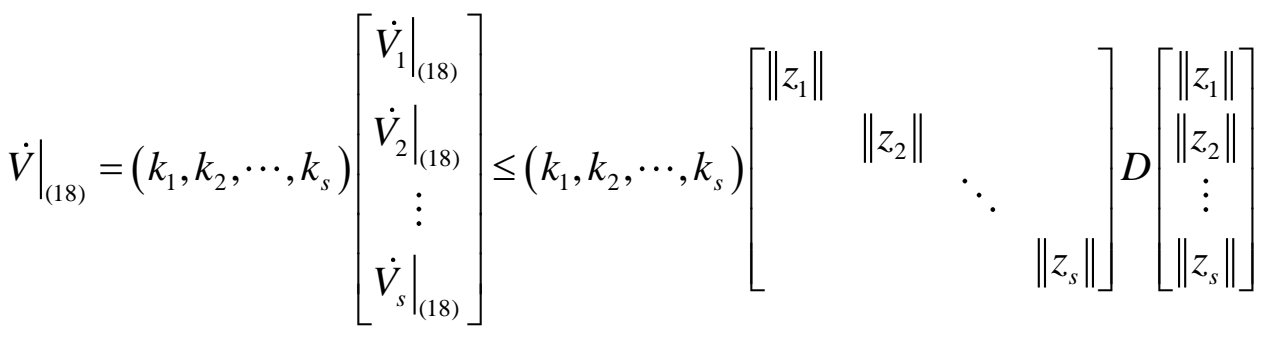

$$
\begin{aligned}
& =\left[\left\|z_{1}\right\|,\left\|z_{2}\right\|, \cdots,\left\|z_{s}\right\|\right]\left(\frac{K D+D^{\mathrm{T}} K}{2}\right)\left[\begin{array}{c}
\left\|z_{1}\right\| \\
\left\|z_{2}\right\| \\
\vdots \\
\left\|z_{s}\right\|
\end{array}\right] .
\end{aligned}
$$

Because matrix $K D+D^{\mathrm{T}} K$ is negative definite, then $\left.\dot{V}\right|_{(18)}$ is negative definite. According to Lyapunov stability theorem, the zero solution of system (18) is (globally) asymptotically stable.

Next, we need to prove $\lim _{t \rightarrow \infty} \theta(t)=0$. Noting that $\dot{r}(t)$ will tend to zero as time goes to infinity (assumption A3), then based on the relationship between $\theta_{i}(t)$ and $g_{i}(t)$, it suffices to prove $\lim _{t \rightarrow \infty} g_{i}(t)=0 \quad(i=1,2, \cdots, s)$. Due to the stability of matrix $\tilde{A}_{c i}^{\mathrm{T}}$, there exist constant scalars $\alpha>0$ and $M>0$ such that $\left\|\exp \left(\sigma \tilde{A}_{c i}^{\mathrm{T}}\right)\right\| \leq M e^{-\alpha \sigma}$. Moreover, it follows from formula (14) that the inequality

$$
\left\|g_{i}(t)\right\| \leq \int_{0}^{l_{r}}\left\|\exp \left(\sigma \tilde{A}_{c i}^{\mathrm{T}}\right)\right\|\left\|P_{i} \tilde{D}_{i}\right\|\|\dot{r}(t+\sigma)\| d \sigma \leq \int_{0}^{l_{r}} M e^{-\alpha \sigma}\left\|P_{i} \tilde{D}_{i}\right\|\|\dot{r}(t+\sigma)\| d \sigma \leq M\left\|P_{i} \tilde{D}_{i}\right\| \int_{0}^{l_{r}}\|\dot{r}(t+\sigma)\| d \sigma
$$

holds, which, together with $\lim _{t \rightarrow \infty} \dot{r}(t)=0$, leads to $\lim _{t \rightarrow \infty} g_{i}(t)=0 \quad(i=1,2, \cdots, s)$ immediately. 
Since the zero solution of system (18) is asymptotically stable and $\lim _{t \rightarrow \infty} \theta(t)=0$, the zero solution of system (16) is asymptotically stable (Chen, 2003). This completes the proof of Theorem 2 .

\section{References}

Araki M (1978) Stability of large-scale nonlinear systems - quadratic-order theory of composite-system method using M-matrices. IEEE Transactions on Automatic Control 23: 129-142.

Bender EK (1968) Optimum linear preview control with application to vehicle suspension. ASME Journal of Basic Engineering 90: 213-221.

Chen WY (2003) On convergent properties for states of non-homogeneous linear systems. Journal of Tianjin University of Light Industry 18: 1-3.

Dashkovskiy SN, Rüffer BS and Wirth FR (2012) Small gain theorems for large scale systems and construction of ISS Lyapunov functions. In: proceedings of the 51st IEEE Conference on Decision and Control 4165-4170.

Duan ZS, Huang L, Wang L and Wang JZ (2004) Some applications of small gain theorem to interconnected systems. Systems \& Control Letters 52: 263-273. 
Duan ZS, Wang JZ, Chen GR and Huang L (2008) Stability analysis and decentralized control of a class of complex dynamical networks. Automatica 44: 1028-1035.

Katayama T and Hirono T (1987) Design of an optimal servomechanism with preview action and its dual problem. International Journal of Control 45: 407-420.

Katayama T, Ohki T, Inoue T and Kato T (1985) Design of an optimal controller for a discrete-time system subject to previewable demand. International Journal of Control 41: 677-699.

Kojima A (2015) $H_{\infty}$ controller design for preview and delayed systems. IEEE Transactions on Automatic Control 60: 404-419.

Kojima A and Ishijima S (2003) $H_{\infty}$ performance of preview control systems. Automatica 39: 693-701.

Labibi B, Lohmann B, Sedigh AK and Maralani PJ (2002) Output feedback decentralized control of large-scale systems using weighted sensitivity functions minimization. Systems \& Control Letters 47(3): 191-198.

Lee HS and Tomizuka M (1996) Robust motion controller design for high-accuracy positioning system. IEEE Transactions on Industrial Electronics 43: 48-55.

Li DM, Hu ZK and Hu HZ (2002) Optimal preview control of linear discrete systems. Journal of Nanjing University of Science and Technology 26: 284-289. 
Liao FC (1985) The stability of a type of linear system with variable coefficients. Journal of University of Science and Technology Beijing 4: 101-106.

Liao FC, Guo YJ and Tang YY (2015) Design of an optimal preview controller for linear time-varying discrete systems in a multirate setting. International Journal of Wavelets Multiresolution and Information Processing 13: 1550050-1-1550050-19.

Liao FC, Takaba K, Katayama T and Katsuura J (2003) Design of an optimal preview servomechanism for discrete-time systems in a multirate setting. Dynamics of Continuous Discrete and Impulsive Systems 5: 727-744.

Liao FC, Tang YY, Liu HP and Wang YJ (2011) Design of an optical preview controller for continuous-time systems. International Journal of Wavelets Multiresolution and Information Processing 9: 655-673.

Liu TF, Hill DJ and Jiang ZP (2011) Lyapunov formulation of ISS cyclic-small-gain in continuous-time dynamical networks. Automatica 47: 2088-2093.

Lunze J (1992) Feedback control of large-scale systems. London: Prentice Hall.

Michel AN and Miller RK (1977) Qualitative analysis of large scale dynamical systems. New York: Academic Press.

Moelja AA and Meinsma G (2006) $H_{2}$ control of preview systems. Automatica 42: 945-952. 
Sheridan TB (1966) Three models of preview control. IEEE Transactions on Human Factors in Electronics 7: 91-102.

Shi L and Singh SK (1992) Decentralized adaptive controller design for large-scale systems with higher order interconnections. IEEE Transactions on Automatic Control 37: 1106-1118.

Tsuchiya T and Egami T (1994) Digital preview and predictive control (translated by Liao Fucheng). Beijing: Beijing Science and Technology Press.

Tomizuka M (1975) Optimal continuous finite preview problem. IEEE Transactions on Automatic Control 20: 362-365.

Tomizuka M (1996) Model based prediction, preview and robust controls in motion control systems. In: proceedings of the 4th international workshop on advanced motion control 1: 1-6.

Zhang $\mathrm{H}$ and Feng G (2008) Stability analysis and $H_{\infty}$ controller design of discrete-time fuzzy large-scale systems based on piecewise Lyapunov functions. IEEE Transactions on Systems Man \& Cybernetics--Part B 38(5): 1390-1401.

Zhang XM and Wang ZL (1996) Research of preview control method on robot. Journal of Beijing University of Aeronautics and Astronautics 22: 21-26.

Zhao L, Sun FQ, Ren JC and Li BW (2016) Optimal preview control for a class of continuous time-invariant descriptor systems. Optimal Control Applications and 
Methods 37: 279-289. 